\title{
Meshless Algorithms for Computational Biomechanics of the Brain
}

\author{
Adam Wittek*, Grand Roman Joldes, Karol Miller \\ Intelligent Systems for Medicine Laboratory, \\ Department of Mechanical Engineering, \\ The University of Western Australia \\ 35 Stirling Highway, Crawley-Perth, 6009 Western Australia, \\ Australia \\ *Email: adam.wittek@uwa.edu.au
}

\section{Introduction}

In Chapter 10, we highlighted two important factors that limit application of finite element method in predicting the brain responses for surgery simulation and pose a significant challenge in application to injury biomechanics when the body tissues undergo rupture/failure:

1) Time consuming generation of patient-specific finite element meshes of the brain and other body organs [1].

2) Deterioration of the solution accuracy and instability when the finite element meshes undergo distortion induced by large localised deformations caused by interactions between the organ and surgical tool [3] and by injury.

Meshless algorithms [4-6], in which the analysed continuum is discretised by nodes (where forces and displacements are calculated) with no assumed structure for the interconnection of the nodes and integration points (where stresses and strains are calculated) (Figure 1 ), have been proposed in the literature for generating computational grids of domains with complex geometry and providing reliable results for large deformations [7-10].

Smoothed particle hydrodynamics $\mathrm{SPH}$ is regarded as the first meshless method. It utilises a strong form of equations of continuum mechanics [12]. SPH and other particle methods (such as material point method in which a strong form of equations of continuum mechanics is used) were applied in injury biomechanics [14-16]. 
However, the literature indicates several important shortcomings of the SPH method, which includes instabilities in tension and accuracy inferior to that of the finite element method [4]. The quest for eliminating this shortcomings and advancing the SPH continues [17] in parallel with development of new algorithms that apply a strong form of equations of solid mechanics [19]. So far, however, such methods have found only limited application in computational biomechanics [20].

Therefore, we focus on meshless methods that utilise the weak form of equations of continuum mechanics and background integration grid. As an example, we discuss the meshless computational biomechanics framework that utilises Total Lagrangian formulation and explicit integration in time domain: Meshless Total Lagrangian Explicit Dynamics (MTLED) [7, 21-26] (Figure 2). Dynamic relaxation and real-time computation of soft tissue deformations through algorithm implementation on Graphics Computing Units (GPUs) discussed in the Chapter 10 for finite element method apply also to this framework.

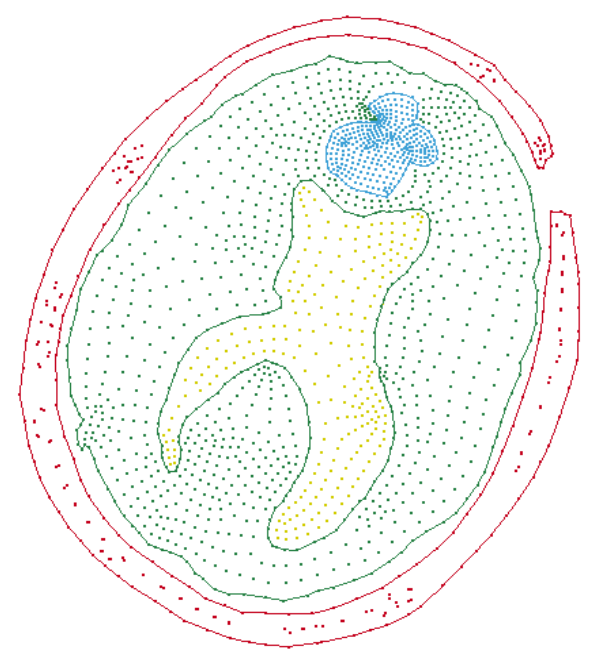

Figure 1 Meshless discretisation (interpolation nodes) of the patientspecific brain geometry for computing deformations within the brain due to the craniotomy induced brain-shift. Adapted from Horton et al. [27]. 


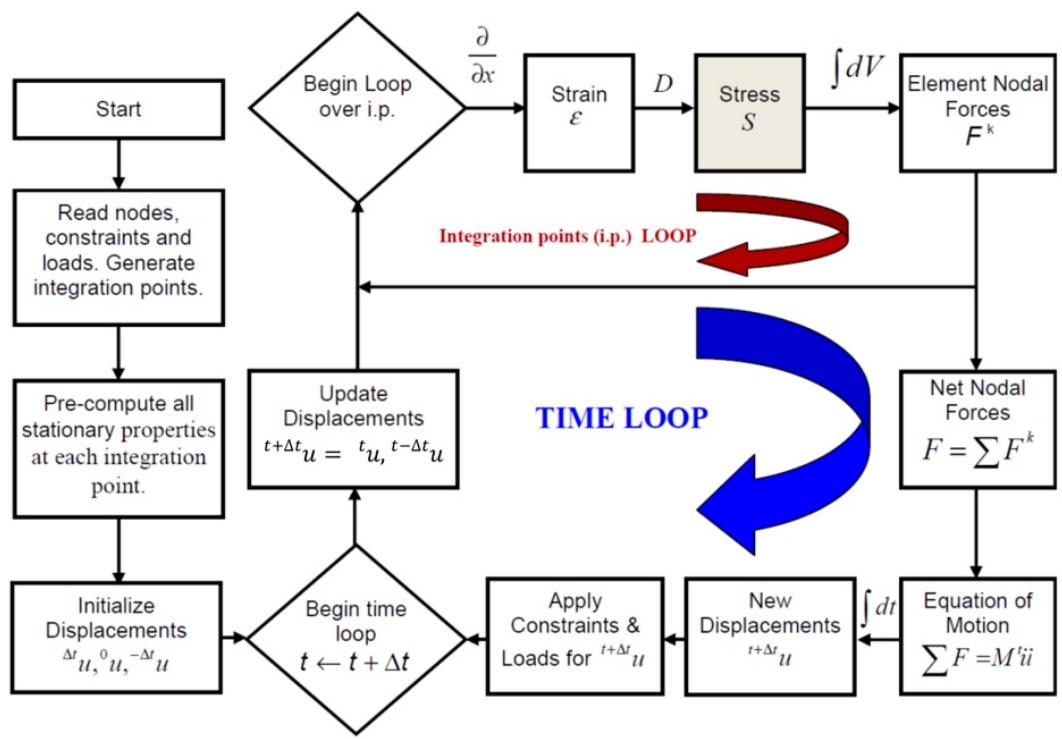

Figure 2 Flowchart of the Meshless Total Lagrangian Explicit Dynamics (MTLED) framework for surgical simulation. Note the important difference with the finite element Total Lagrangian Explicit Dynamics (TLED) algorithm discussed in Chapter 10. In the MTLED framework, the spatial discretisation is done over a cloud of points and the spatial integration is done using the background grid. Therefore, unlike in the TLED, the loop is over the integration points rather than the elements.

The key motivation for Meshless Total Lagrangian Explicit Dynamics (MLTED) framework is the need for computational biomechanics simulations to satisfy the constraints and requirements of neurosurgical navigation. This includes fast creation of patientspecific (representing a given patient) computational biomechanics models and conducting surgical simulations without the requirement for the user to become an expert in computational mechanics (as hospitals are unlikely to hire PhDs in computational mechanics to do surgery planning). In the MTLED framework, we propose to achieve this through introducing specialised shape functions and adaptive spatial integration that facilitate accurate solution even if the analysed 
continuum is discretised using irregularly/non-uniformly distributed nodes, and through the specialised algorithm that employs the visibility criterion for surgical dissection and tissue rupture simulation. Therefore, in the subsequent sections of this Chapter, we discuss the following topics:

- Section 2: Shape functions for meshless algorithms for computing soft tissue deformations;

- Section 3: Spatial integration schemes for meshless algorithms for computing soft tissue deformations;

- Section 4: Visibility criterion for modelling of surgical dissection and soft tissue rupture;

- Section 5: Stability of the specialised meshless explicit dynamics algorithm for surgical simulation;

- Section 6: Algorithm verification.

\section{Shape Functions for Meshless Algorithms for Computing Soft Tissue Deformations}

The method for interpolation/approximation of the displacement field and the type of shape functions used for such interpolation are some of the crucial differences between the MTLED framework and the Total Lagrangian Explicit Dynamics (TLED) finite element algorithm described in Chapter 10. The TLED, and vast majority of finite element algorithms, use polynomial shape functions. In the MTLED framework, we use Moving Least-Squares shape functions that were initially proposed by Lancaster and Salkuaskas [28] for approximation of scattered data and later applied by Nayroles et al. [29] in the Diffuse Element Method:

$$
u^{h}(\mathbf{x})=\mathbf{p}^{\mathbf{T}}(\mathbf{x}) \mathbf{a}(\mathbf{x}),
$$

where $u^{h}$ is the approximation of the displacement $u, \mathbf{p}(\mathbf{x})$ is the vector of monomial basis function, $\mathbf{a}(\mathbf{x})$ is the vector of coefficients that need to be calculated, and $\mathbf{x}$ is the point belonging to the analysed continuum but not located at the node. In the MTLED framework, low order (up to quadratic order) monomial basis functions are used 
[7]:

$$
\mathbf{p}^{\mathrm{T}}(\mathbf{x})=\left(1|x y z| x y x z y z \mid x^{2} y^{2} z^{2}\right) .
$$

The coefficients $a(\mathbf{x})$ are computed by minimising an error functional $J$ defined based on the weighted least squares errors for $n$ points located at positions $x_{j}(j=1, \ldots, n)$ :

$$
J(\mathbf{x})=\sum_{j=1}^{n}\left[\left(u^{h}\left(\mathbf{x}_{j}\right)-u_{j}\right)^{2} w\left(\left\|\mathbf{x}-\mathbf{x}_{j}\right\|\right)\right],
$$

where $w$ is the weight function (positive weight function is used) and $\|\cdot\|$ denotes the Euclidean distance. Given Eq. (1), the error functional $J$ (defined in Eq. 3) can be rewritten as [30]

$$
J=(\mathbf{P a}-\mathbf{u})^{\mathbf{T}} \mathbf{W}(\mathbf{P a}-\mathbf{u}),
$$

where

$$
\begin{aligned}
\mathbf{u}^{\mathbf{T}} & =\left[\begin{array}{lll}
u_{1}, u_{2} & \ldots & u_{n}
\end{array}\right], \\
\boldsymbol{P} & =\left[\begin{array}{cccc}
p_{1}\left(\mathbf{x}_{1}\right) & p_{2}\left(\mathbf{x}_{1}\right) & \cdots & p_{m}\left(\mathbf{x}_{1}\right) \\
p_{1}\left(\mathbf{x}_{2}\right) & p_{2}\left(\mathbf{x}_{2}\right) & \cdots & p_{m}\left(\mathbf{x}_{2}\right) \\
\vdots & \vdots & \ddots & \vdots \\
p_{1}\left(\mathbf{x}_{\mathrm{n}}\right) & p_{2}\left(\boldsymbol{x}_{n}\right) & \cdots & p_{m}\left(x_{n}\right)
\end{array}\right], \\
\boldsymbol{W} & =\left[\begin{array}{cccc}
w\left(\left\|\mathbf{x}-\mathbf{x}_{1}\right\|\right) & 0 & \cdots & 0 \\
0 & w\left(\left\|\mathbf{x}-\mathbf{x}_{2}\right\|\right) & \cdots & 0 \\
\vdots & \vdots & \ddots & \vdots \\
0 & 0 & \cdots & w\left(\left\|\mathbf{x}-\mathbf{x}_{n}\right\|\right)
\end{array}\right],
\end{aligned}
$$

where $u_{i}$ is the value of the field variable (displacement) at node $i$. To minimise the error functional Jgiven in Eq. (4), it's partial derivatives $\frac{\partial J}{\partial \mathbf{a}}$ are set to zero [30]

$$
\frac{\partial J}{\partial \mathbf{a}}=\mathbf{P}^{\mathrm{T}} \mathbf{W} \mathbf{P a}(\mathbf{x})-\mathbf{P}^{\mathrm{T}} \mathbf{W} \mathbf{u}=0
$$

and the coefficients $\mathbf{a}(\mathbf{x})$ at the evaluation points are obtained as

$$
\mathbf{a}(\mathbf{x})=\left(\mathbf{P}^{\mathrm{T}} \mathbf{W} \mathbf{P}\right)^{-1} \mathbf{P}^{\mathrm{T}} \mathbf{W} \mathbf{u},
$$

where $\mathbf{P}^{\mathrm{T}} \mathbf{W P}$ is also referred to as the moment matrix $\mathbf{M}=\mathbf{P}^{\mathrm{T}} \mathbf{W} \mathbf{P}$. 
Following Joldes et al. [30], from Eq. (9), the shape functions $\boldsymbol{\Phi}$ can be defined as:

$$
\boldsymbol{\Phi}(\mathbf{x})=\left[\phi_{1}(\mathbf{x}) \ldots \phi_{n}(\mathbf{x})\right]=\mathbf{P}^{\mathrm{T}}\left(\mathbf{P}^{\mathrm{T}} \mathbf{W} \mathbf{P}\right)^{-\mathbf{1}} \mathbf{P}^{\mathrm{T}} \mathbf{W} .
$$

According to Eq. (10), the shape functions can be constructed only if the moment $\mathbf{M}=\mathbf{P}^{\mathrm{T}} \mathbf{W P}$ is non-singular. This implies that although the requirements regarding the nodal distribution are much less stringent that in the Finite Element Method, where the nodes need to be arranged in a mesh of high-quality tetrahedral or hexahedral elements (see Chapter 10), some conditions still apply and not all the nodal distributions are acceptable/admissible. These conditions depend on the bases of the shape functions. This poses a challenge for meshless algorithms for surgical simulation as the end-users are medical professionals rather than experts in computational mechanics and, due to complex geometry of the brain and other body organs, irregular nodal distributions are an effective and convenient approach for spatial discretisation [31, 32]. Application of such distributions makes it possible to generate patient-specific computational biomechanics models of the brain and other organs directly from images [32, 33, 34] (Figure 3). However, unlike in the case of the finite element method discussed in Chapter 10, there are no specialised computational grid generators for the MTLED framework and other meshless methods using weak form of equations of continuum mechanics. In the examples showed in Figure 3, Figure 4, Figure 7 and Figure 8, the nodes were created using the established algorithms available in the HyperMesh ${ }^{\mathrm{TM}}$ finite element preprocessing software package (by Altair, MI, USA; https://altairhyperworks.com/product/hypermesh). The process is similar to automated generation of nodal distributions for tetrahedral finite element meshes, but as no elements need to be constructed, the usual constraints on the node placement due to element quality considerations disappear.

We require from the shape functions in the MTLED framework to facilitate robust approximation for irregular nodal distributions without the need for the user to control/adjust the parameters of the distributions to achieve accurate solution. Modified Moving Least shape functions introduced by Joldes et al. [30] and Chowdhury et al. [25] address this challenge for higher order bases functions. 


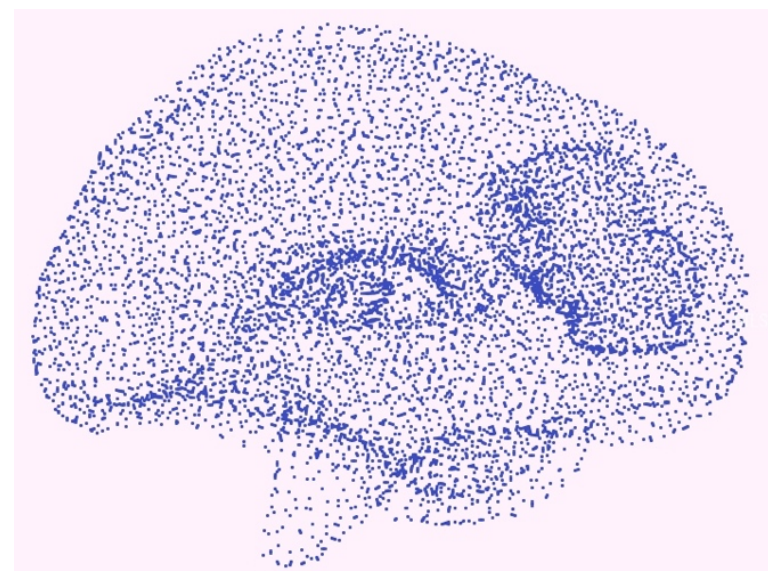

Figure 3 Irregular nodal distribution applied in Miller et al. [31] for computing the deformations within the brain due to craniotomyinduced brain shift.

The key idea behind the Modified Moving Least Square (MMLS) function comes from the realisation that the singularity of the moment matrix $\mathbf{M}=\mathbf{P}^{\mathrm{T}} \mathbf{W} \mathbf{P}$ in Eq. (8) originates from the fact that Eq. (9), applied for computing the coefficients $\mathbf{a}(\mathbf{x})$, has multiple solutions. This implies that functional $J$ (Eq. 4) does not include sufficient constraints to guarantee a unique solution for a given nodal distribution. Therefore, to prevent singularities for the second order bases functions, we add additional constraints to the functional $J[30]$ :

$$
J(\mathbf{x})=\sum_{j=1}^{n}\left[\left(u^{h}\left(\mathbf{x}_{j}\right)-u_{j}\right)^{2}+\mu_{x^{2}} a_{x^{2}}^{2}+\mu_{x y} a_{x y}^{2}+\mu_{y^{2}} a_{y^{2}}^{2}\right],
$$

where

$$
\boldsymbol{\mu}=\left[\begin{array}{lll}
\mu_{x^{2}} & \mu_{x y} & \mu_{y^{2}}
\end{array}\right]
$$

is the vector of positive weights for the additional constraints. From Eq. (4), that defines the functional for MLS shape functions, and Eq. (11), a new functional $\bar{J}$ for (new) MMLS shape functions can be rewritten as:

$$
\bar{J}=(\mathbf{P a}-\mathbf{u})^{\mathrm{T}} \mathbf{W}(\mathbf{P a}-\mathbf{u})+\mathbf{a}^{\mathrm{T}} \mathbf{H a},
$$

where $\mathrm{H}$ is the matrix with all elements $\mathbf{0}_{33}$ equal to zero except the 
last three diagonal entries that are equal to the weights $\mu$ of the additional constraints (see Eq. 12):

$$
H=\left[\begin{array}{cc}
\mathbf{0}_{33} & \mathbf{0}_{33} \\
\mathbf{0}_{33} & \operatorname{diag}(\boldsymbol{\mu})
\end{array}\right] .
$$

To compute the coefficients $\mathbf{a}(\mathbf{x})$ for the MMLS shape functions, we minimise the functional $\bar{J}$ (Eq. 13) following the procedure previously used for MLS (as given by Eq. 8, Eq. 9 and Eq. 10). This leads to the following formula for computing (new) MMLS shape functions [30]:

$$
\overline{\boldsymbol{\Phi}}(\mathbf{x})=\left[\bar{\phi}_{1}(\mathbf{x}) \ldots \bar{\phi}_{n}(\mathbf{x})\right]=\mathbf{P}^{\mathrm{T}}\left(\mathbf{P}^{\mathrm{T}} \mathbf{W} \mathbf{P}+\mathbf{H}\right)^{-1} \mathbf{P}^{\mathrm{T}} \mathbf{W} .
$$

This formula differs from that for the traditional MLS functions (Eq. 10) by the constraints weight matrix $\mathrm{H}$. The constraints are to prevent singularities in the error functional $\bar{J}$ (Eq. 11, Eq. 12 and Eq. 13).

In has been indicated in Chowdhury et al. [25] and Joldes et al. [30] that the MMLS shape functions appreciably improve accuracy of prediction of the brain deformations due to surgery in comparison to the traditionally used MLS functions (Figure 4) and provide solution for irregular nodal distributions where the MLS functions exhibit singularity. 
a)

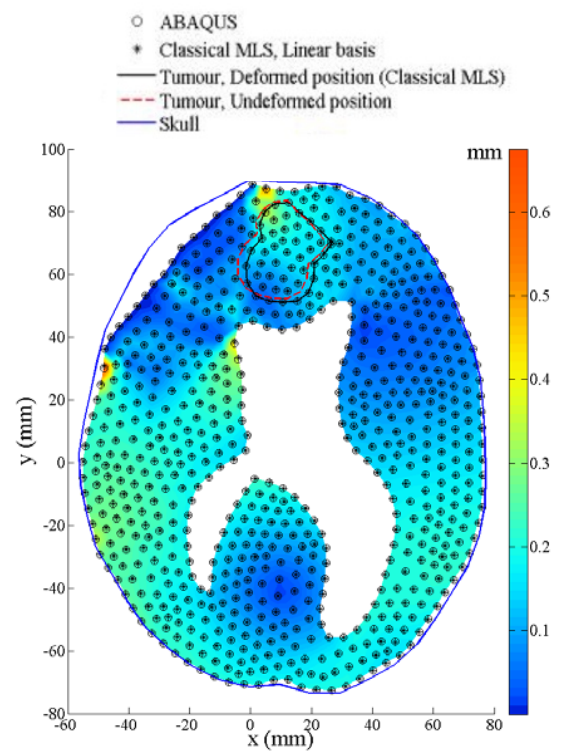

b)

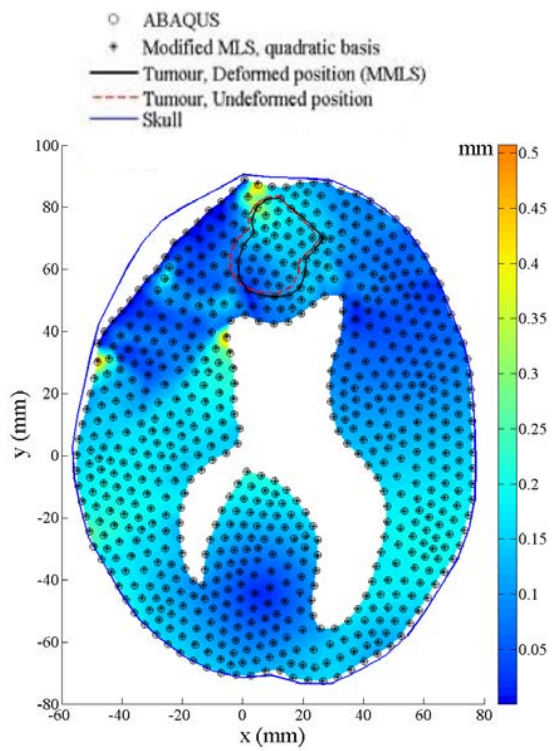

Figure 4 Evaluation of the Meshless Total Lagrangian Explicit Dynamics (MTLED) framework with traditionally used Moving Least Square (MLS) shape functions and Modified Moving Least Square (MMLS) shape functions introduced by Joldes et al. [30] and Chowdhury et al. [25]. The evaluation is conducted through application in predicting the brain deformations due to craniotomyinduced brain shift. a) Differences between the deformations predicted using the MTLED with MLS shape functions and wellestablished non-linear finite element code ABAQUS [35]; b) Differences between the deformations predicted using the MTLED with MMLS shape functions and well-established non-linear finite element code ABAQUS. The solution obtained using ABAQUS finite element code is regarded as the reference. Accuracy improvement due to application of MMLS shape functions is clearly visible. Adapted from Chowdhury et al. [36]. 


\section{Spatial Integration Schemes for Meshless Algorithms for Computing Soft Tissue Deformations}

Two main types of integration schemes are used in the meshless methods of computational mechanics:

1) Gaussian quadrature $Q$ over a background grid where the integration is done over the integration cells $D$ with one or more integration points per cell [7, 24, 37, 38]:

$$
\begin{aligned}
& I=\int_{D} f(\mathbf{x}) d D, \\
& I \cong Q_{n}(D)=\sum_{i=1}^{n} w_{i} f\left(\mathbf{x}_{i}\right),
\end{aligned}
$$

where $f$ is the function we intend to integrate, $I$ is the integral approximated using the $n$-point Gaussian quadrature $Q_{n}$ over the integration cell $D, \mathbf{x}_{i}$ are the integration points, and $w_{i}$ are the corresponding weights.

2) Nodal integration where the interpolating nodes are also used as integration points $[39,40]$.

As the literature indicates that Gauss quadrature over the background integration cells tends to be less computationally demanding than nodal integration schemes [42], in the Meshless Total Lagrangian Explicit Dynamics (MTLED) framework we use the background integration (Figure 5).

In the MTLED framework (and other meshless algorithms that rely on weak formulation of equations of continuum mechanics), application of background integration using Gaussian quadrature is associated with errors that originate from two sources:

1) Shape functions in meshless methods are not polynomials [42];

2) Shape functions' support may not align with the integration cells.

Difficulty in estimation and control of such errors is a common challenge for many of the existing integration schemes [24]. 


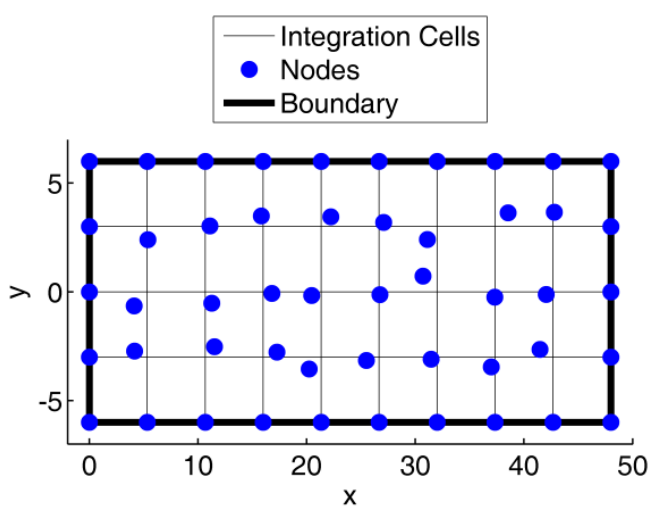

Figure 5 Meshless discretisation of the problem domain by an irregular nodal distribution with a background grid of quadrilateral integration cells. Adapted from Joldes et al. [44].

In 2011 edition of this book [43], we advocated hexahedral cells with a single integration point per cell (the idea similar to the one used in underintegrated hexahedral elements discussed in Chapter 5) (Figure 6). As integration cells do not have to conform to the boundary of the analysed continuum, they can be generated automatically even for complex geometry. The nodes, where the displacements are calculated, are independent of the background integration grid [7]. Simplicity and flexibility are key advantages of spatial integration using hexahedral background grids. Almost arbitrary placement of the nodes throughout the analysed continuum can be used, which is well suited for complex geometry of the brain and other human body organs. However, restrictions on the ratio of the number of integration points and nodes apply. Through parametric study, we estimated in in Horton et al. [7] that the number of integration points should be twice the number of nodes for accurate and convergent solution. Although we successfully used this estimate in predicting the deformations within the brain due to craniotomyinduced brain shift [31] (Figure 7), it provides only very limited control of the integration error. Consequently, the analyst's knowledge of meshless methods of computational mechanics and experience in using them are crucial for ensuring accuracy of the computations. This poses a challenge for clinical applications where the users are medical professionals rather than experts in 
computational mechanics. To address this challenge, we incorporated into the MTLED framework an adaptive integration scheme that adapts the quadrature to the behaviour of the function being integrated $[24,44]$.

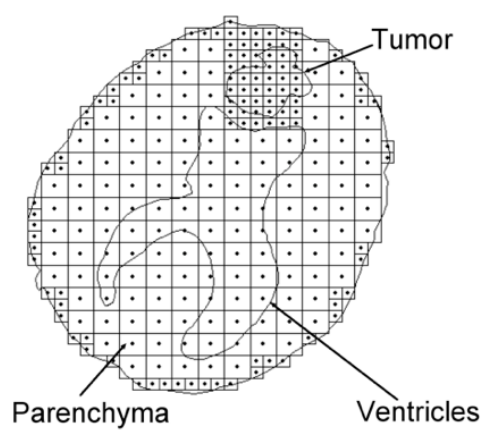

Figure 6 Background regular integration grid for a patient-specific meshless model of the brain with tumour. The integration points are indicated as $(\cdot)$. Note that the background grid does not conform to the geometry boundary. Adapted from Horton et al. [27].

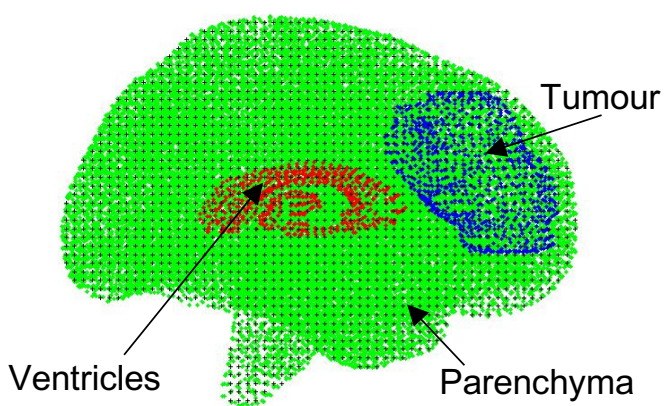

Figure 73 -D patient-specific meshless model for computing the deformations within the brain due to craniotomy-induced brain shift for image-guided neurosurgery. The model was implemented using the Meshless Total Lagrangian Explicit Dynamics MTLED framework. Detailed description and the results obtained using this model are in Miller et al. [31]. The integration points are indicated as $(+)$ and interpolating nodes - as $\left({ }^{\circ}\right)$ for the brain parenchyma, $\left({ }^{\circ}\right)$ for the ventricles, and $\left({ }^{\circ}\right)$ for the tumour. Regular hexahedral background integration grid (similar to that shown for two-dimensional 2-D model in Figure 6) was used. 
For the Meshless Total Lagrangian Explicit Dynamics (MTLED) framework, we proposed an adaptive integration scheme where the integration cells $D$ (see Eq. 16 and Eq. 17) are recursively subdivided into $m$ smaller cells until the desired integration tolerance $\tau$ is achieved [24, 44]. Using notation $Q_{n}^{m}(D)$ to indicate the $n$-point quadrature applied on $m$ subdivided regions of the integration cell $D$, our adaptive integration scheme (as proposed in [24, 44]) can be described using the following pseudo-code:

\section{Adaptive Integration Scheme}

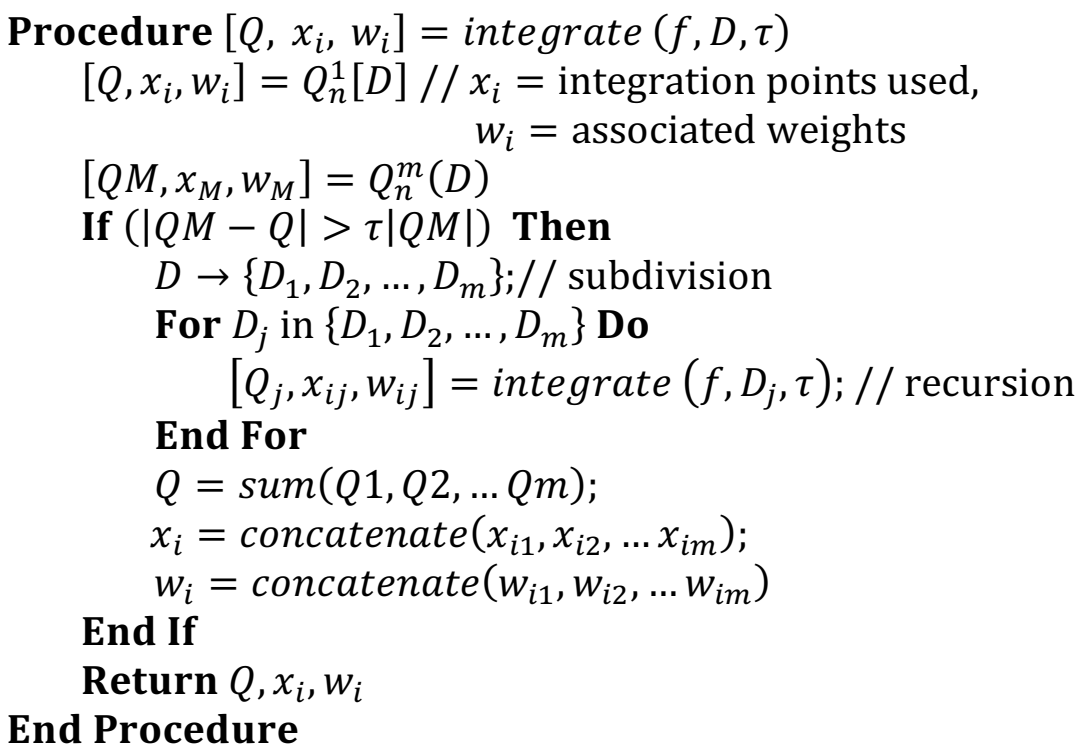

The above integration scheme has the following properties that make it very attractive for application in computational biomechanics of the brain and other body organs:

- The size of the integration cells is automatically adjusted (the number of integration cells in the areas where the shape functions exhibit large variations is automatically increased to maintain the integration accuracy); 
- New integration points are introduced only in the areas where the integration accuracy is below the desired (required) accuracy;

- The scheme works for any shape and size of support domains;

- The scheme is particularly effective for irregular/non-uniform nodal distributions.

In practice, defining the relative integration tolerance $\tau$ is the only input required from the analyst for the adaptive integration scheme used in the MTLED framework. Although it is rather difficult to formulate detailed guidelines regarding selection of such tolerance, the numerical experiments we conducted in Joldes et al. [24] have indicated that the solution accuracy does not appreciably increase after the integration accuracy reaches a certain level/threshold. This, in turn, suggests that it is not necessary to use very high integration accuracy (low tolerance $\tau$ ) resulting in many subdivisions of each integration cell.

We recommend to conduct a convergence analysis to determine the best integration accuracy for a given spatial discretisation. Such analysis has been conducted by Joldes et al. [44] for the problem of computation of the brain deformations due to craniotomy induced brain shift (Figure 8). In such problems, the required solution accuracy is within the voxel size of the intraoperative Magnetic Resonance (MR) images - between $1 \mathrm{~mm}$ and $2 \mathrm{~mm}$. The results obtained by Joldes et al. [44] suggest that such accuracy of prediction of the brain deformations can be achieved using the relative integration tolerance $\tau \leq 0.1$ (i.e. 0.1 is the maximum acceptable tolerance) (Figure 8). 
a)

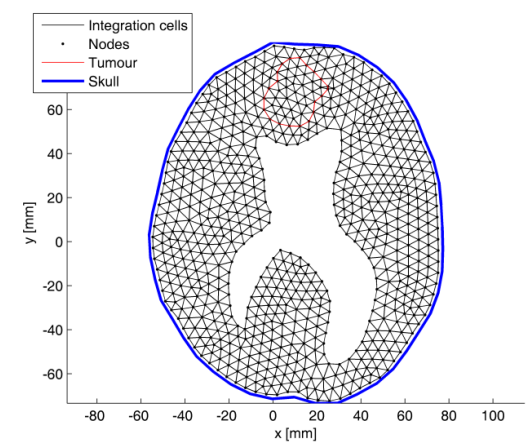

b)

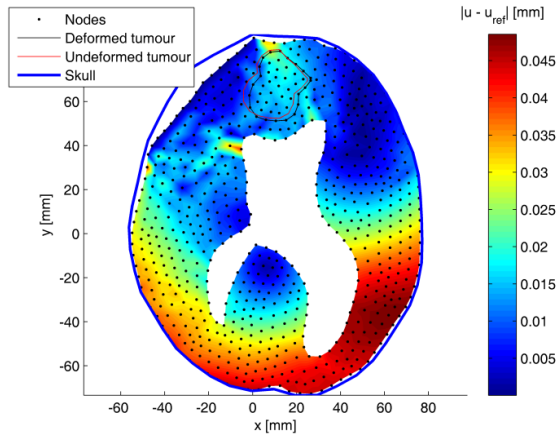

Figure 8 Application of the MTLED framework with the adaptive integration scheme in predicting the brain deformations due to craniotomy-induced brain shift. The figure shows the influence of the integration tolerance on the solution (predicted brain deformations) accuracy. a) Meshless discretisation using nodes and triangular background integration cells. The nodes define vertices of the cells. b) Differences between the deformations predicted using three Gauss points per integration cell and the reference solution using very stringent relative integration tolerance $\tau=0.001$. For the tolerance $\tau=0.1$, the maximum difference with the reference solution is around $0.035 \mathrm{~mm}$. As this difference is well within the required accuracy of $1 \mathrm{~mm}$ (less than half of the voxel size of a typical intraoperative Magnetic Resonance Image MRI), we recommend tolerance $\tau$ slightly below 0.1 Adapted from Joldes et al. [44].

\section{Visibility Criterion for Modelling of Surgical Dissection and Soft Tissue Rupture}

Surgical dissection and injury-related rupture introduce discontinuities/cracks in the body tissues and organs. In the computational biomechanics algorithms that rely on finite element discretisation discussed in Chapter 10, surgical dissection is simulated by subdividing the elements forming the mesh [45-49]. This requires sophisticated re-meshing techniques to generate new elements with good aspect ratio and map the field variables from the original to the new mesh. With an exception of Bui et al. [49], the re-meshing is in 
practice limited to tetrahedral elements that exhibit volumetric locking when applied to soft tissues and other incompressible continua unless special countermeasures are applied (see Chapter 10). Furthermore, error accumulation due to re-meshing tends to constrain the accuracy of finite element method in modelling of surgical cutting/dissection $[47,50]$. As the meshless methods utilise spatial discretisation in a form of a "cloud" of points/nodes, the burden associated with re-meshing required by finite element method is to large extend alleviated. Therefore, meshless discretisation has been proposed by several authors as a method of choice for modelling of continua undergoing crack propagation and fragmentation [8, 51-57].

The specialised algorithm, created for the MTLED framework by Jin et al. [23] at the Intelligent Systems for Medicine Laboratory at The University of Western Australia, models the progressive surgical cutting by adding and/or splitting nodes on the cutting path using the visibility criterion. The visibility criterion prevents the nodes located on the opposites side of dissection/crack from interacting with each other (they are "invisible" to each other) $[4,58]$. In the algorithm by Jin et al. [23], the surgical cut and injury-caused rupture/crack are geometrically represented using a series of line segments with the aid of the level set method [59,60] to mathematically describe the location of all the nodes and integration points in relation to the cutting/rupture path (Figure 9). The effect of cutting/rupture induced discontinuity is entirely reflected in the changes of the shape and size of the nodal influence domains (Figure 10). 
Cutting Line L: $\psi=0$

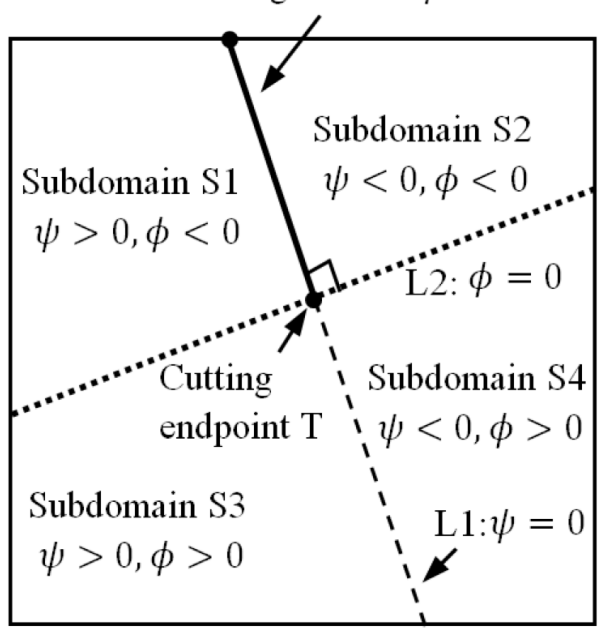

Figure 9 Specialised visibility for modelling dissection and rupture in the MTLED framework. The cutting/crack direction is represented as the zero level set of function $\psi(x, y)=\left(x-x_{e p}\right) \frac{v_{y}}{\|\mathbf{V}\|}-(y-$ $\left.y_{e p}\right) \frac{v_{x}}{\|\mathbf{V}\|}$, where $(x, y)$ is the coordinate of a given point in the problem domain; $\left(x_{e p}, y_{e p}\right)$ is the coordinate of the end-point of the cutting/crack path; $V_{x}$ and $V_{y}$ are the components of vector $\mathbf{V}$ representing the cutting/crack direction; and $\|\mathbf{V}\|$ is the length of vector V. The end-point of the cut/crack is represented as the intersection of the zero level set of function $\psi(x, y)$ with the orthogonal zero level set of function $\varphi(x, y)=\left(x-x_{e p}\right) \frac{v_{x}}{\|\mathbf{V}\|}-$ $\left(y-y_{e p}\right) \frac{v_{y}}{\|\mathbf{v}\|}$. The domain is divided into four subdomains according to the sign of level sets of functions $\psi$ and $\varphi$. Division into these four subdomains is used to determine the position of a point and supporting node in relation to the cutting/crack line L. Adapted from Jin et al. [23]. 
Cutting line $\mathrm{L}: \psi=0$

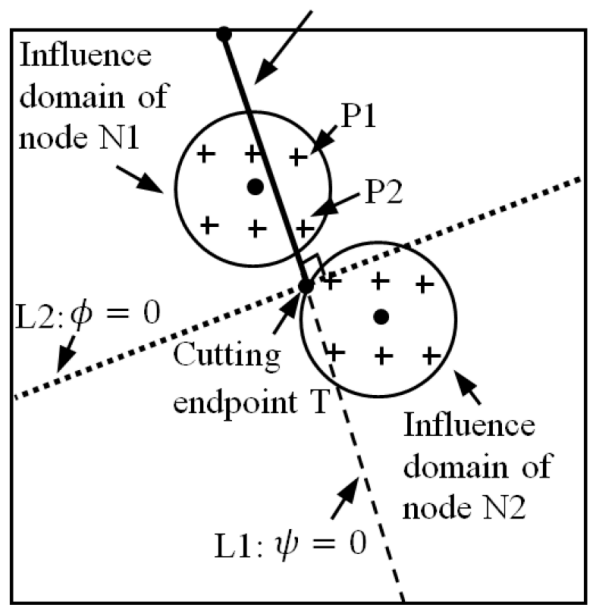

Figure 10 Specialised visibility criterion for modelling dissection and rupture in the Meshless Total Lagrangian Explicit Dynamics MTLED framework. The effect of cutting/rupture induced discontinuity is entirely reflected in the changes of the shape and size of the nodal influence domain. The influence domain of node N1 intersects the cutting/crack line $\mathrm{L}$; points $\mathrm{P} 1$ and $\mathrm{P} 2$ are eliminated from the influence domain of this node. The influence domain of node N2 passes through the cutting endpoint $\mathrm{T}$ only, so it does not need an update. Adapted from Jin et al. [23].

Quantitative evaluation through application in modelling of rupturecausing elongation of the specimen of pia-arachnoid complex has confirmed the robustness and accuracy of the specialised visibility criterion for modelling dissection and rupture in MTLED framework [18] [23] (Figure 11). However, application in modelling of dissection of 3-D continua indicated challenges associated with high computational cost of the visibility criterion and level set method in 3-D [61]. Therefore, we recommend visibility criterion only for modelling of dissection and rupture of thin tissue layers such as the brain meninges. To be best of our knowledge, despite ongoing research effort that includes application of the methods such as the phase-field approach [62], the problem of modelling of crack propagation in 3-D continuum subjected to large deformations and 
exhibiting non-linear stress-strain relationship still awaits solution that can be regarded as accurate (in a sense of quantitatively accurate predictions of forces and deformations), robust, computationally efficient and suitable for application in surgery simulation where the users are unlikely to have expert knowledge of computational mechanics. Therefore, we offer no recommendation regarding the method of choice for 3-D computational biomechanics modelling of surgical dissection and rupture propagation.

a)

b)

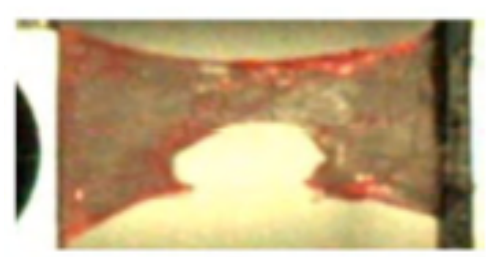

c)

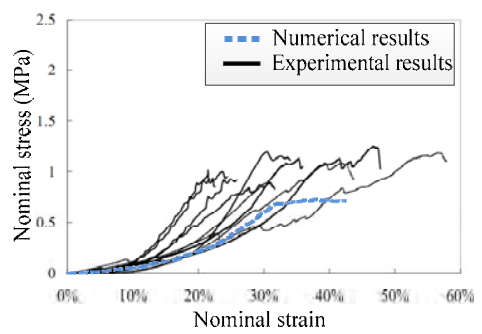

Figure 11 Quantitative evaluation of the performance of the Meshless Total Lagrange Explicit Dynamics (MTLED) framework with the specialised visibility criterion for modelling surgical cutting/dissection and soft tissue rupture. The results obtained when applying the MTLED framework to model rupture-causing elongation of pia-arachnoid-complex tissue specimen are compared with the experimental data obtained by Jin et al. [2] at the Bioengineering Center, Wayne State University. The strain rate is $0.05 \mathrm{~s}^{-1}$. a) The specimen deformation for the overall sample elongation of $42.5 \%$ of the initial length predicted using the MTLED framework. Dimensions are in $\mathrm{mm}$; b) Photograph of the deformed tissue specimen. Adapted from Jin [11] (Bioengineering Center, Wayne State University). c) Comparison of the nominal stress-strain relationship predicted using the MTLED framework [18] with the experimental results reported in [2]. The figures and the photograph are adapted from [11] and [18]. 


\section{Stability of Explicit Dynamics Meshless Algorithms For Computation of Soft Tissue Deformation}

Both the Meshless Total Lagrangian Explicit Dynamics (MTLED) framework discussed here and the Total Lagrangian Explicit Dynamics (TLED) finite element algorithm for neurosurgical simulation described in Chapter 10, use the central difference method for time stepping. This method is only conditionally stable. The critical time step $\Delta t_{\text {crit }}$ that ensures the computation stability can be determined from the maximum frequency of vibrations $\omega_{\max }$ (or the maximum eigenvalue $A_{\max }$ ) of a given system (as represented by the model) [63]:

$$
\Delta t_{\text {crit }}=\frac{2}{\omega_{\max }}=\frac{2}{\sqrt{A_{\max }}} .
$$

In Chapter 10, when discussing the TLED finite element algorithm, we stated that it can be demonstrated from Gerschgorin's theorem $[64,65]$ that the maximum eigenvalue of an assembled finite element mesh is bounded by the maximum eigenvalue of any of the elements in the mesh. Consequently, for the TLED algorithm, we obtain the maximum eigenvalue of the analysed system $A_{\max }$ by estimating the maximum eigenvalue of each element in the mesh $\lambda_{\text {max }}^{e}$. In Joldes et al. [66], we applied this reasoning to the MTLED framework by replacing the element eigenvalues with the eigenvalues $\lambda_{\max }^{I}$ for the integration points. This leads to the following conservative estimate of critical time step $\Delta t_{\text {crit }}$ for the MTLED framework [66]:

$$
\Delta t_{\text {crit }}=\frac{2}{\omega_{\max }}=\frac{2}{\sqrt{A_{\max }}} \approx \frac{2}{\sqrt{\max _{I}\left(\lambda_{\max }^{I}\right)}}=\min _{I}\left(\frac{2}{\sqrt{\lambda_{\max }^{I}}}\right) .
$$

Following Bathe [67], the maximum eigenvalue $\lambda_{\max }^{I}$ for a given integration point $I$ can be estimated from the Rayleigh quotient as

$$
\lambda_{\max }^{I}=\sup _{\mathbf{u}} \frac{\mathbf{u}^{T} \mathbf{K}^{I} \mathbf{u}}{\mathbf{u}^{T} \mathbf{M}^{I} \mathbf{u}}
$$

where $\mathbf{K}^{I}$ is the stiffness matrix for the integration point $I, \mathbf{u}$ is the vector of nodal displacements for the nodes associated with the 
integration point $I$, and $\mathbf{M}^{I}$ is the mass matrix for the integration point I.

In both, the finite element TLED algorithm described in Chapter 10 and in the MTLED framework discussed here, lumped mass matrices are used. Physical interpretation of such matrices is that the system mass is assigned entirely (lumped) to the nodes. In the MTLED framework, the mass allocated to the integration point $I$ is distributed equally to all nodes within the support domain of that integration point [66]. Therefore, Eq. (20) can be rewritten as [66]:

$$
\lambda_{\text {max }}^{I}=\frac{N^{I}}{m^{I}} \sup \frac{\mathbf{u}^{T} \mathbf{K}^{I} \mathbf{u}}{\mathbf{u}^{T} \mathbf{u}}=\frac{N^{I}}{m^{I}} \rho_{\max }\left(\mathbf{K}^{I}\right),
$$

where $N^{I}$ is the number of nodes in the support domain of the integration point $I, \mathrm{~m}^{I}$ is the mass allocated to the integration point $I$, and $\rho_{\max }\left(\mathbf{K}^{I}\right)$ is the maximum eigenvalue of the stiffness matrix $\mathbf{K}^{I}$ for the integration point $I$.

The stiffness matrix is defined in terms of the straindisplacement matrices $\mathbf{B}^{I}$ for a given integration point $I$, elasticity matrix $\mathbf{C}$ that contains the information about the constitutive properties, and volume $V^{I}$ allocated to the integration point $I[66,68]$ :

$$
\mathbf{K}_{i j l K}^{I}=\mathbf{B}_{j J}^{I}\left(\mathbf{x}^{I}\right) \mathbf{C}_{i j k l} \mathbf{B}_{l K}^{I}\left(\mathbf{x}^{I}\right) \cdot V^{I},
$$

where the subscripts indicate the tensor order, i.e. $i j k l$ and $i J l k$ indicate the fourth order tensor, $j J$ and $l K-$ the second order tensor. For the homogenous materials with the constitutive properties defined using Lame constants $(\lambda$ and $\mu$ ), the maximum eigenvalue of the stiffness matrix $\rho_{\max }\left(\mathbf{K}^{I}\right)$ for the integration point $I$ can be estimated as [68]:

$$
\rho_{\max }\left(\mathbf{K}^{I}\right) \leq(\lambda+\mu) \cdot V^{I} \cdot\left\|\mathbf{B}^{I}\right\|_{F}^{2},
$$

where $\left\|\mathbf{B}^{I}\right\|_{F}$ is Frobenius norm $\left\|\mathbf{B}^{I}\right\|_{F}^{2}=\mathbf{B}_{j I} \mathbf{B}_{j I}$. Substituting Eq. (23) into Eq. (21) leads to the following formulae for the upper bounds of the maximum eigenvalues of the stiffness matrix for the integration point $I[66]$ :

$$
\lambda_{\max }^{I} \leq N^{I}\left(c^{I}\right)^{2} \cdot \mathbf{B}_{j I}^{I} \mathbf{B}_{j I}^{I},
$$


where $c$ is the dilatational (acoustic) wave speed. By substituting Eq. (24) into Eq. (18), the critical time step $\Delta t_{\text {crit }}$ for the MTLED framework can be conservatively estimated as:

$$
\Delta t_{\text {crit }} \approx \operatorname{Min}_{I}\left(\frac{2}{\sqrt{N^{I}\left(c^{I}\right)^{2} \cdot \mathbf{B}_{j I}^{I} \mathbf{B}_{j I}^{I}}}\right) .
$$

The formula for determining the bounds for maximum eigenvalue $\lambda_{\text {max }}^{I}$ of the stiffness matrix given in Eq. (24) is valid also for the finite element method, as long as the same mass lumping scheme is used. Therefore, it applies also to the 8-noded hexahedral element with a single integration point we discussed in Chapter 10 as the element of choice for computational biomechanics of the brain.

\section{Algorithm Verification}

We follow the verification approach introduced in Chapter 10 where the results obtained by means of new algorithms of non-linear computational mechanics are compared with the solutions from the established algorithms. However, none of the existing weak-form meshless methods of computational mechanics has been recognised by the research community as a gold-standard yet. Therefore, following Chapter 10, also in this Chapter, we use the results obtained from the established algorithms implemented in commercial nonlinear finite element codes as a reference solution.

In the following sections we present verification results for some of the algorithms described in this Chapter: Meshless Total Lagrangian Explicit Dynamics (MTLED) framework, Modified Moving Least Square (MMLS) shape function for deformation interpolation, and specialised visibility criterion for modelling surgical dissection/tissues rupture. 


\subsection{Meshless Total Lagrangian Explicit Dynamics (MTLED) framework}

The Meshless Total Lagrangian Explicit Dynamics (MTLED) framework has been verified by comparing the results obtained using this framework with the established finite element code (ABAQUS implicit dynamics non-linear solver [35]) was used when modelling semi-confined uniaxial compression and shear of a cylinder made from a very soft (shear modulus of $1 \mathrm{kPa}$ ) hyperelastic (neo-Hookean) material. In the meshless discretisation of the cylinder almost arbitrary node placement and hexahedral integration cells nonconforming to the geometry were used (Figure 12).
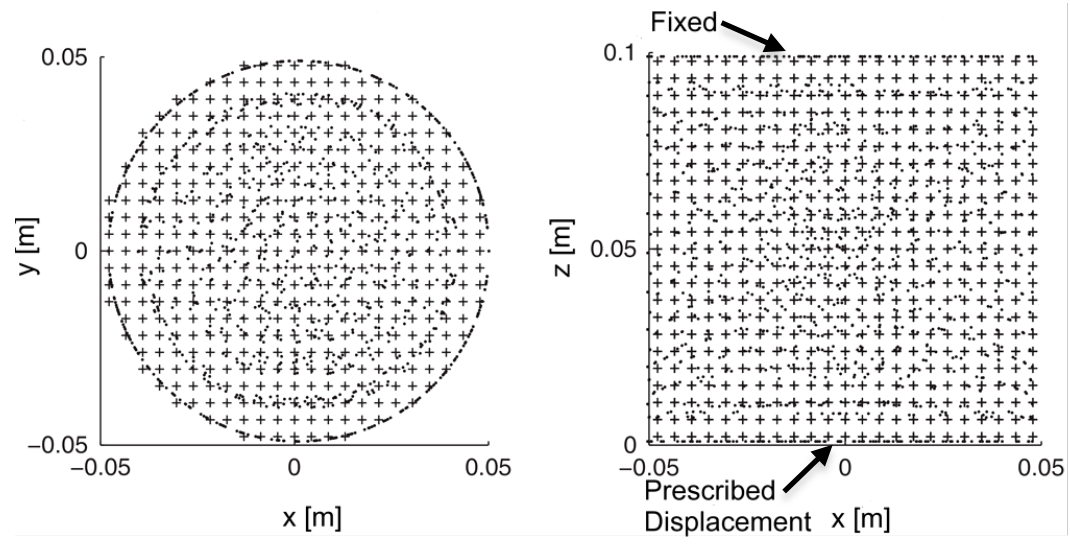

Figure 12 Meshless model of a cylinder used in verification of the MTLED algorithm by Horton et al. [7]. The nodes are indicated as (.) and integration points as (+). Note almost arbitrary node placement. The integration points do not conform to geometry. The boundary conditions are shown in the right-hand-side figure: the nodes on the top boundary were constrained and the prescribed displacement was applied to the nodes on the bottom boundary. Adapted from Horton et al. [7].

For $20 \%$ compression and shear of the cylinder, the difference in the total reaction force on the displaced cylinder surface between MTLED framework and ABAQUS implicit finite element solver [35] was no more than 5\% (Figure 13a). The force-time histories obtained using the meshless framework were qualitatively similar to 
those from the finite element method. The maximum relative difference in the computed deformations between the MTLED framework and ABAQUS was around 3.5\% (it can be seen in Figure $13 \mathrm{~b}$ that some of the nodes in meshless discretisation do not sit exactly on the deformed finite element boundary).

a)
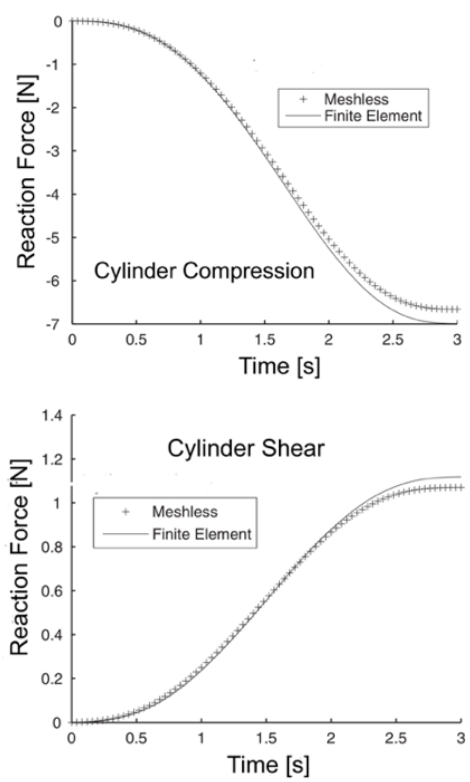

b)
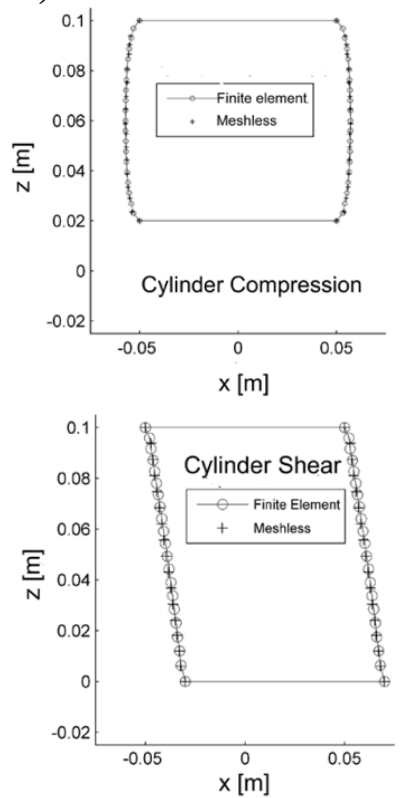

Figure 13 Comparison of the results obtained when modelling $20 \%$ compression and shear of a cylinder using meshless (MTLED framework) and finite element (ABAQUS implicit solver [35]) discretisation. a) Reaction force - time histories; b) Contour of the deformed cylinder at time of $3 \mathrm{~s}$. The displacement $u$ was enforced over a period $T=3 \mathrm{~s}$ using a 3-4-5 polynomial that ensures zero velocity and acceleration at time $t=0$ and time $t=T$ [69]. The displacement magnitude was $0.02 \mathrm{~m}$ in $z$ direction for compression and $0.02 \mathrm{~m}$ in $x$ direction for shear. $x$ and $z$ directions are defined in Figure 12. Adapted from Horton et al. [7].

The MTLED framework produces stable results even for very large deformations as indicated by the energy - time histories obtained when modelling the cylinder compressed to $20 \%$ of it's original height (nominal compressive strain of 0.8) (Figure 14). For 
such large compression no verification against the ABAQUS finite solver could be done as the finite element solution became unstable. This is also demonstrated by recent results obtained when comparing the performance of the MTLED framework with the non-linear finite procedures available in ABAQUS solver in modelling of indentation of cylindrical samples made from soft incompressible material (Sylgard 527 silicone gel by Dow Corning) with properties similar to the brain tissue (Figure 15a) [70]. The MTLED framework facilitates the solution for the indentation depth of $59 \%$ of the sample initial height while the finite element procedures become unstable (the solution "fails") for the indentation depth of only $24 \%$ of the sample initial height (Figure 15b and 15c).

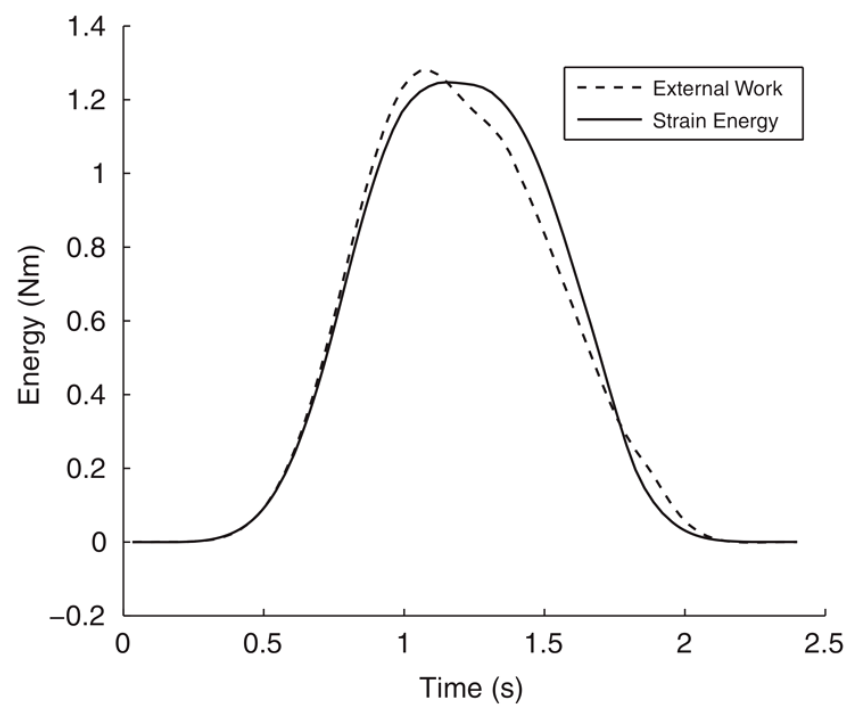

Figure 14 MTLED algorithm. External work and strain energy when compressing a cylinder to $20 \%$ of its original height (and returning to the initial state). The displacement was enforced using a 3-4-5 polynomial [69]. Adapted from Horton et al. [7]. 
a)

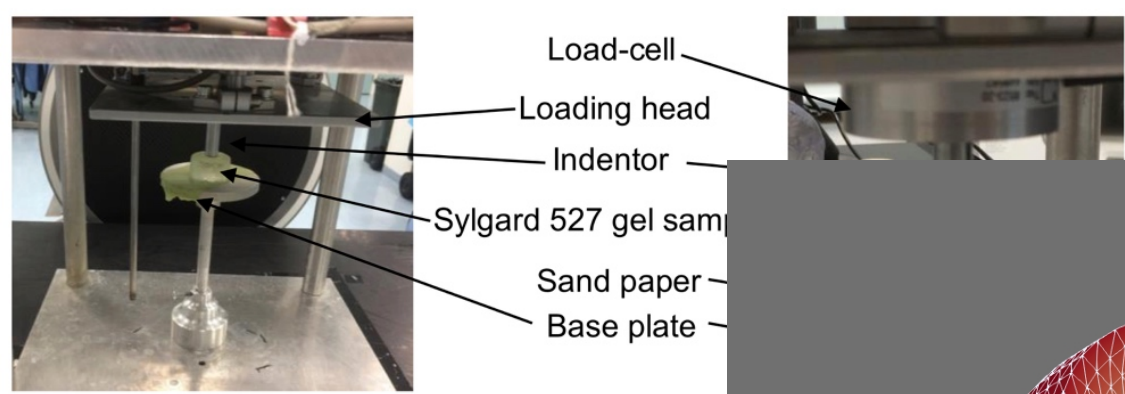

b)

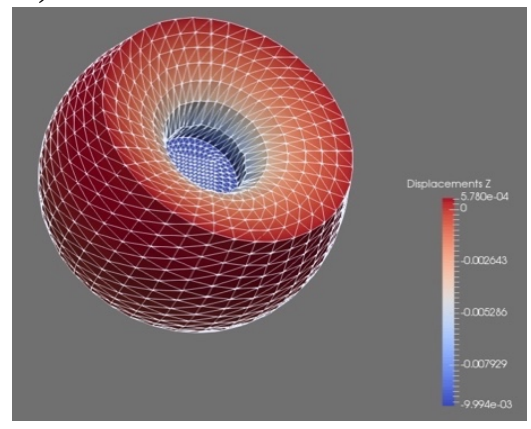

c)

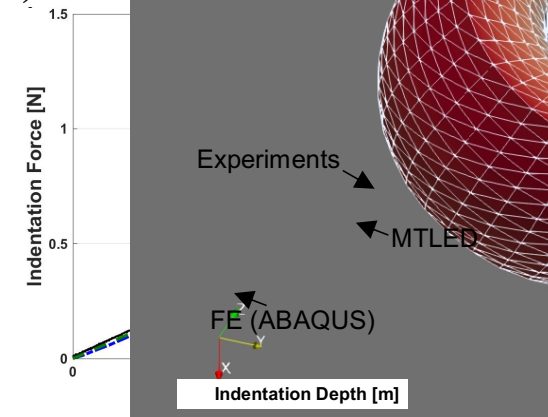

Figure 15 Verification of the MTLED algorithm through modelling of indentation of cylindrical samples made from soft incompressible material (Sylgard 527 silicone gel by Dow Corning) with properties similar to the brain tissue. a) Experimental set-up. b) Overall deformation of the meshless model for the indentation depth of 10 $\mathrm{mm}$ (the displacement scale in the figure is in meters). The interpolating nodes were connected to form the triangles to visualise the deformed model surface. c) Comparison of the force - indentation depth relationship obtained using the MTLED framework (green dotted line), non-linear finite element procedures available in ABAQUS finite element (FE) code (blue dotted line), and the experimental data (black solid line) - average from three experiments. Using ABAQUS finite element code [35] we were able to obtain the results for the indentation depth of up to only $4 \mathrm{~mm}$ after which the solution diverged. For the indentation depth of up to $4 \mathrm{~mm}$, the results obtained using the MTLED framework and ABAQUS code are very close and cannot be visually distinguished. Adapted from Wittek et al. [70]. 


\subsection{Modified Moving Least Square (MMLS) shape functions for computing soft tissue deformation}

We verified the performance of the MTLED framework with Modified Moving Least Square (MMLS) shape functions trough application in modelling of an extension of a 2-D rectangular specimen (dimensions $10 \mathrm{~cm} \times 4 \mathrm{~cm}$ ) (Figure 16) [30]. The results obtained using the MTLED framework were compared with wellestablished non-linear finite element code (ABAQUS static nonlinear solver [35] with default configuration was used). Detailed description is provided in Joldes et al. [30]. The rectangular specimen was discretised using 57 nodes. To ensure the integration accuracy, a dense regular background integration grid was used. It consisted of 4000 rectangular integration cells with a single integration (Gauss) point per cell. The nodes on the left-hand-side edge of the specimen were rigidly constrained while the displacement of $3 \mathrm{~cm} \mathrm{(30 \%} \mathrm{of} \mathrm{the}$ initial specimen length) was applied to the nodes on the right-handside edge (Figure 16a). In the finite element model implemented using the ABAQUS code, the spatial discretisation was done using 57 nodes and 84 four-noded rectangular elements.

As reported in Joldes et al. [30], the results indicate a very good agreement between the results obtain using the MTLED framework with MMLS shape functions and ABAQUS static non-linear finite element solver - the maximum difference in the computed deformations was under $0.5 \mathrm{~mm}$ (Figure 16b). They also show appreciable accuracy improvement in comparison to the traditionally used Moving Least Square (MLS) shape functions. However, it should be noted that as the resolution of intraoperative (acquired during surgery) Magnetic Resonance Images (MRIs) and accuracy of state-of-the-art neurosurgery techniques is not better than $1 \mathrm{~mm} \mathrm{[71],}$ even the accuracy achieved using MLS can be confidently regarded as sufficient for application in image-guided neurosurgery. 
a)

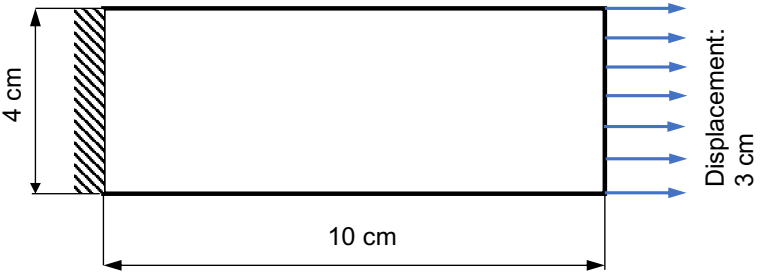

b)

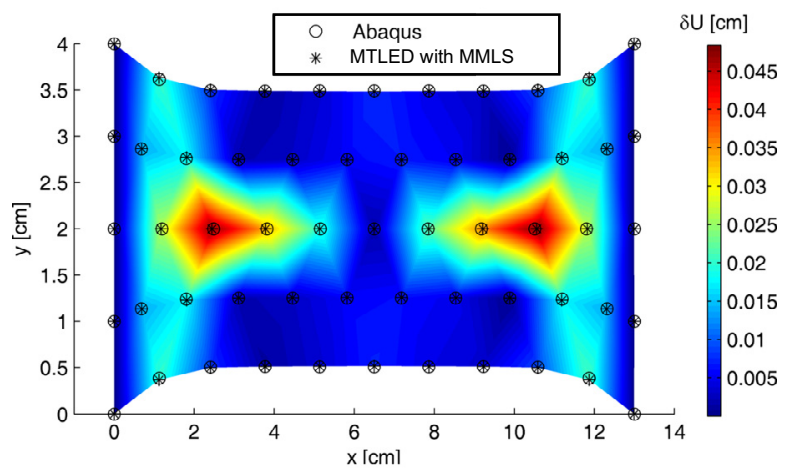

Figure 16 Verification of the Modified Moving Least Square (MMLS) shape functions implemented in the MTLED framework through application in modelling of elongation of a rectangular specimen with the constitutive properties consistent with the brain tissue. a) Geometry and boundary conditions for the model. b) Differences in the computed deformations between the MTLED framework with MMLS shape functions and ABAQUS static nonlinear finite element solver. Adapted from Joldes et al. [30]. 


\subsection{Visibility criterion for modelling of surgical dissection and soft tissue rupture}

Verification of visibility criterion for modelling of surgical dissection and soft tissue rupture was conducted through application in simulation of dissection of a rectangular (dimensions $100 \mathrm{~mm} \times 100$ $\mathrm{mm}$ ) specimen of soft hyperelastic material undergoing elongation of $20 \%$ of the initial length (Figure 17). We used the neo-Hookean hyperelastic constitutive model [72], with the parameters (Young's modulus of $E=3000 \mathrm{~Pa}$, Poisson's ratio of $v=0.49$, mass density of $\rho=1000 \mathrm{~kg} / \mathrm{m}^{3}$ ) consistent with the brain tissue constitutive properties.

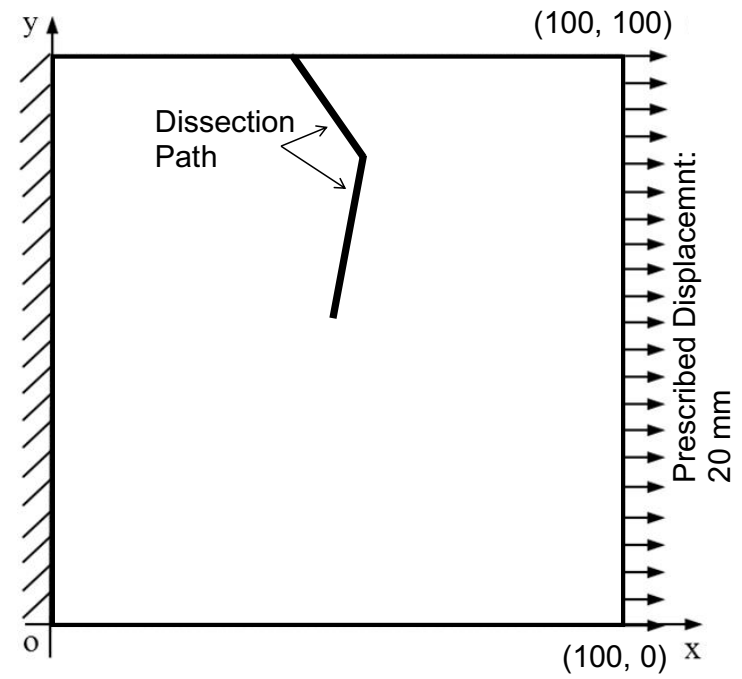

Figure 17 Geometry for the model for verification of visibility criterion implemented in the Meshless Total Lagrangian Explicit Dynamics (MTLED) framework for modelling of surgical dissection and soft tissue rupture. The verification was conducted through application in simulation of dissection of a rectangular specimen of soft incompressible material with the brain tissue constitutive properties undergoing elongation of $20 \%$ of the initial length. Adapted from Jin et al. [23].

As discussed in Chapter 10, simulation of surgical dissection using the finite element method is associated with a number of formidable challenges/difficulties. They include deterioration of the 
solution accuracy when the elements forming the mesh become distorted under large deformations and the need for re-meshing to introduce a crack/discontinuity due to dissection/rupture and as a mesh distortion countermeasure. Therefore, when obtaining the reference solution for verification of the MTLED framework with the visibility criterion using the established non-linear procedures available in the ABAQUS finite element code [35], surgical dissection could not be directly modelled. Instead, the elongation was applied to the finite element model of a specimen of soft hyperelastic material (with the brain tissue properties) with a pre-defined dissection/cut. The edges of the elements were aligned and separated along the dissection (Figure 18). The analysis using the ABAQUS finite element code was conducted for implicit integration in time domain with the default parameters and linear (i.e. with linear shape functions) quadrilateral plain strain elements with hybrid formulation to prevent volumetric locking. It was confirmed through $\mathrm{H}$ refinement (mesh density increase) that the finite element discretisation (using 7183 nodes) used in this analysis provides a converged solution. The meshless model implemented using the MTLED framework consisted of 6151 nodes (the discretisation density was confirmed using convergence analysis) (Figure 19).

As the distribution and position of nodes in the meshless model implemented using the MTLED framework and finite element model implemented using the ABAQUS code were different, the nodal displacements obtained using the MTLED framework were recalculated (through interpolation using the MLS shape functions) for the nodal positions of the model implemented using the ABAQUS code to enable verification of the predicted deformations. 


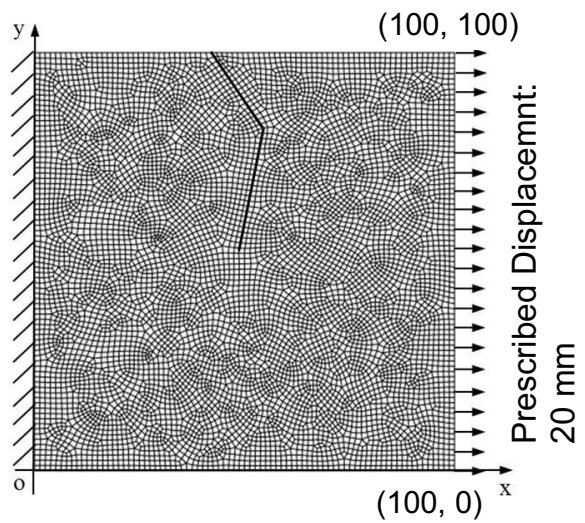

Figure 18 The initial configuration of the finite element model with a pre-defined dissection/cut implemented using ABAQUS non-linear finite element code. The results obtained using this model were used a reference when verifying the Meshless Total Lagrangian (MTLED) framework with visibility criterion for modelling of surgical dissection/soft tissue rupture. Dimensions are in millimetres (mm). Adapted from Jin et al. [23].

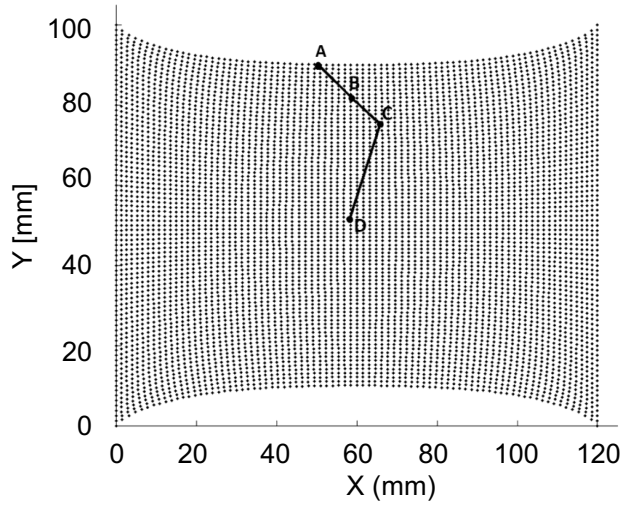

Figure 19 Meshless model for verification of the Meshless Total Lagrangian Explicit Dynamics (MTLED) framework with visibility criterion for modelling of surgical dissection and tissue rupture. Spatial discretisation was done using 6151 nodes. In this model, the dissection was carried out in the stretched specimen of brain tissuelike material along the pre-defined path indicated using thick line segments. Dimensions are in millimetres $(\mathrm{mm})$. Adapted from Jin et al. [23] . 
The verification results indicate a very good agreement between the internal forces and deformations predicted using the MTLED framework with visibility criterion and the reference finite element solution obtained using ABAQUS non-linear code (a static procedure was used) (Figure 20). The relative differences for the reaction force are only of $0.5 \%$. The maximum absolute difference in the predicted deformations is $0.5 \mathrm{~mm}(2.56 \%$ of the imposed elongation) and the average difference (averaging over all model nodes) is only $0.03 \mathrm{~mm}$ ( $0.15 \%$ of the imposed elongation). Therefore, considering that the accuracy of state-of-art neurosurgery techniques is not better than 1 mm [71], Meshless Total Lagrangian Explicit Dynamics (MTLED) framework with visibility criterion for surgical dissection modelling can be regarded as satisfying the accuracy requirements of computerintegrated surgery.

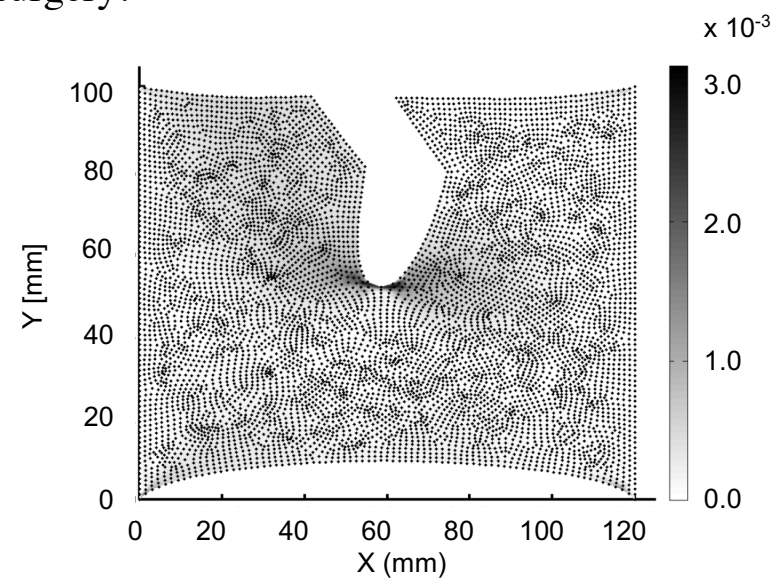

Figure 20 Results of verification of Meshless Total Lagrangian Explicit Dynamics (MTLED) framework with visibility criterion for modelling of surgical dissection and tissue rupture: deformed meshless model implemented using the MTLED framework with the dissection modelled using visibility criterion. The figure shows the absolute difference between the deformation magnitudes computed using the MTLED framework and the reference results from the established non-linear static solution procedures available in the ABAQUS finite element code. The dimensions and deformations are in mm. Adapted from Jin et al. [23]. 


\section{Conclusions}

The field of surgery simulation is dominated by finite element analysis. However, time consuming generation of patient-specific finite element meshes (computational grids) and deterioration of the solution accuracy when the elements undergo distortion induced by large deformations due to surgery remain a formidable challenge that prevents computational biomechanics to become a part of surgical training and planning workflow [1]. For more than 10 years, we have advocated meshless methods of computational mechanics, in which the computational grid has the form of a "cloud" of points, as one possible solution to overcome this challenge [1, 7, 27, 31,32]. Based on our experience with both strong (Smoothed Particle Hydrodynamics SPH and finite difference-collocation method) [16, 19] and weak [1, 7, 27, 31, 32] formulations of meshless methods of computational mechanics, for computational biomechanics of the brain, we recommend the weak formulation with background cells for spatial integration [7, 24]. We have used such integration in the Meshless Total Lagrangian Explicit Dynamics MTLED framework described in this Chapter [7]. The MTLED framework retains all the advantages associated with the explicit stepping in time domain as discussed in Chapter 10 in the context of finite element method. They include: no need for iterations even for non-linear problems, no need to solve a system of equations, very modest internal memory requirements, and suitability for parallel processing implementation.

We view meshless methods of computational mechanics not only as algorithms for computing the responses of soft tissues and body organs undergoing large deformations and fragmentation (due to surgical dissection and injury) but primarily as a framework that would enable an analyst (medical professional) who is not an expert in computational mechanics to create patient-specific computational biomechanics models of the brain and apply them in surgery simulation with a guarantee of numerical accuracy of the results. Modified Moving Least Square (MMLS) shape functions [30] and adaptive integration introduced [24] in the Meshless Total Lagrangian Explicit Dynamics framework are steps in this direction. They ensure accuracy and robustness of solution of the equations of continuum mechanics for irregular/non-homogenous nodal distributions that 
facilitate automated discretisation of the complex geometry of the brain and creation of computational grids directly from neuroimages [33]. Defining solution tolerance is the only input they require from the user.

The visibility criterion $[18,23]$ discussed and recommended in this Chapter in the context of simulation of surgical dissection and injury-related tissue rupture leads to high computational cost when applied to three-dimensional dissection/rupture propagation [61]. Therefore, development of meshless algorithms that facilitate efficient and robust modelling of surgical dissection and injuryrelated rupture of soft tissues provides the next challenge in surgery and injury simulation.

\section{Acknowledgements}

This Chapter uses the published results of research supported by the funding from the Australian Government through the Australian Research Council ARC (Discovery Project Grants DP160100714, DP1092893, and DP120100402) and National Health and Medical Research Council NHMRC (Project Grants APP1006031 and APP1144519). The Authors thank Prof. King Hay Yang and Dr Xin Jin of Bioengineering Center and Biomedical Engineering Department of Wayne State University for providing the experimental data used in the reference [18]. 


\section{References}

1. Wittek, A., Grosland, N., Joldes, G., Magnotta, V., Miller, K.: From Finite Element Meshes to Clouds of Points: A review of methods for generation of computational biomechanics models for patient-specific applications. Annals of Biomedical Engineering 44, 3-15 (2016)

2. Jin, X., Lee, J.B., Leung, L.Y., Zhang, L., Yang, K.H., King, A.I.: Biomechanical response of the bovine pia-arachnoid complex to tensile loading at varying strain-rates. Stapp Car Crash Journal 50, 637-649 (2006)

3. Wittek, A., Dutta-Roy, T., Taylor, Z., Horton, A., Washio, T., Chinzei, K., Miller, K.: Subject-specific non-linear biomechanical model of needle insertion into brain. Computer Methods in Biomechanics and Biomedical Engineering 11, 135-146 (2008)

4. Belytschko, T., Krongauz, Y., Organ, D., Fleming, M., Krysl, P.: Meshless methods: An overview and recent developments. Computer Methods in Applied Mechanics and Engineering 139, 3-47 (1996)

5. Li, S., Liu, W.K.: Meshfree and particle methods and their applications. Applied Mechanics Reviews 55, 1-34 (2002)

6. Sukumar, N., Dolbow, J., Devan, A., Yvonnet, J., Chinesta, F., Ryckelynck, D., Lorong, P., Alfaro, I., Martínez, M.A., Cueto, E., Doblaré, M.: Meshless methods and partition of unity finite elements. International Journal of Forming Porcesses 8, 409-427 (2005)

7. Horton, A., Wittek, A., Joldes, G.R., Miller, K.: A meshless Total Lagrangian explicit dynamics algorithm for surgical simulation. International Journal for Numerical Methods in Biomedical Engineering 26, 977-998 (2010)

8. Doblare, M., Cueto, E., Calvo, B., Martinez, M.A., Garcia, J.M., Cegonino, J.: On the employ of meshless methods in biomechanics. Computer Methods in Applied Mechanics and Engineering 194, 801-821 (2005)

9. Horton, A., Wittek, A., Miller, K.: Subject-specific biomechanical simulation of brain indentation using a meshless method, Lecture Notes in Computer Science 4791, 541-548 (2007)

10. Zhang, G.Y., Wittek, A., Joldes, G.R., Jin, X., Miller, K.: A three-dimensional nonlinear meshfree algorithm for simulating mechanical responses of soft tissue. Engineering Analysis with Boundary Elements 42, 60-66 (2014)

11. Jin, X.: Biomechanical response and constitutive modeling of bovine piaarahcnoid complex. pp. 140. Wayne State University, United States -Michigan (2009)

12. Monaghan, J.J.: Smoothed particle hydrodynamics. Annual Review of Astronomy and Astrophysics 30, 543-574 (1992) 
13. Lee, S.-H., Darvish, K., Lobovsky, L.: Fluid-structure interaction in finite element modeling of traumatic aortic rupture. In: Proceedings of the ASME 2004 International Mechanical Engineering Congress and Exposition, 337338. ASME Paper No. IMECE2004-61790, ASME (2004)

14. Ionescu, I., Guilkey, J., Berzins, M., Kirby, R.M., Weiss, J.: Computational simulation of penetrating trauma in biological soft tissues using the material point method. Studies in Health Technology and Informatics 111, 213-218 (2005)

15. Hieber, S.S.E.: Remeshed smoothed particle hydrodynamics simulation of the mechanical behavior of human organs. Technology and Health Care 12, 305314 (2004)

16. Wittek, A., Omori, K., Nakahira, Y.: Effects of brain-skull boundary conditions on responses of simplified finite element brain model under angular acceleration in sagittal plane. In: Proceedings of the Mechanical Engineering Congress, Tokushima, Japan. 101-102, Japan Society of Mechanical Engineers (2003)

17. Maurel, B., Combescure, A., Potapov, S.: A robust SPH formulation for solids European Journal of Computational Mechanics 15, 495-512 (2006)

18. Jin Xia, Zhang, G., Joldes, G.R., Yang, K.-H., Jin Xin, Rohan, P.-Y., Miller, K., Wittek, A.: 2-D Meshless algorithm for modelling of soft tissue undergoing fragmentation and large deformation:Verification and performance evaluation. In: Proceedings of the 10th International Symposium on Computer Methods in Biomechanics and Biomedical Engineering, 327 332, Arup (2012)

19. Bourantas, G.C., Mountris, K.A., Loukopoulos, V.C., Lavier, L., Joldes, G.R., Wittek, A., Miller, K.: Strong-form approach to elasticity: Hybrid finite difference-meshless collocation method (FDMCM). Applied Mathematical Modelling 57, 316-338 (2018)

20. Toma, M.: The emerging use of SPH in biomedical applications. Significances of Bioengineering \& Biosciences 1, 1-4 (2017)

21. Zhang, Y.J., Joldes, G.R., Wittek, A., Miller, K.: Patient-specific computational biomechanics of the brain without segmentation and meshing. International Journal for Numerical Methods in Biomedical Engineering 29, 293-308 (2013)

22. Zhang, G.Y., Wittek, A., Joldes, G.R., Jin, X., Miller, K.: A three-dimensional nonlinear meshfree algorithm for simulating mechanical responses of soft tissue. Engineering Analysis with Boundary Elements 42, 7 (2013)

23. Jin, X., Joldes, G.R., Miller, K., Yang, K.H., Wittek, A.: Meshless algorithm for soft tissue cutting in surgical simulation. Computer Methods in Biomechanics and Biomedical Engineering 17, 800-817 (2014) 
24. Joldes, G.R., Wittek, A., Miller, K.: Adaptive numerical integration in Element-Free Galerkin methods for elliptic boundary value problems. Engineering Analysis with Boundary Elements 51, 52-63 (2015)

25. Chowdhury, H.A., Wittek, A., Miller, K., Joldes, G.R.: An Element Free Galerkin Method Based on the Modified Moving Least Squares Approximation. Journal of Scientific Computing 71, 1197-1211 (2017)

26. Joldes, G.R., Chowdhury, H., Wittek, A., Miller, K.: A new method for essential boundary conditions imposition in explicit meshless methods. Engineering Analysis with Boundary Elements 80, 94-104 (2017)

27. Horton, A., Wittek, A., Miller, K.: Towards meshless methods for surgical simulation. In: Proceedings of the Computational Biomechanics for Medicine Workshop, Medical Image Computing and Computer-Assisted Intervention MICCAI 2006, 34-42 (2006)

28. Lancaster, P., Salkauskas, K.: Surfaces generated by Moving Least-Squares methods. Mathematics of Computation 37, 141-158 (1981)

29. Nayroles, B., Touzot, G., Villon, P.: Generalizing the finite element method: Diffuse approximation and diffuse elements. Computational Mechanics 10, 307-318 (1992)

30. Joldes, G.R., Chowdhury, H.A., Wittek, A., Doyle, B., Miller, K.: Modified moving least squares with polynomial bases for scattered data approximation. Applied Mathematics and Computation 266, 893-902 (2015)

31. Miller, K., Horton, A., Joldes, G.R., Wittek, A.: Beyond finite elements: A comprehensive, patient-specific neurosurgical simulation utilizing a meshless method. Journal of Biomechanics 45, 2698 - 2701 (2012)

32. Li, M., Miller, K., Joldes, G.R., Kikinis, R., Wittek, A.: Biomechanical model for computing deformations for whole-body image registration: A meshless approach. International Journal for Numerical Methods in Biomedical Engineering 32, e02771-02718 (2016)

33. Zhang, J.Y., Joldes, G.R., Wittek, A., Miller, K.: Patient-specific computational biomechanics of the brain without segmentation and meshing. International Journal for Numerical Methods in Biomedical Engineering 29, 293-308 (2013)

34. Heye, Z., Linwei, W., Hunter, P.J., Pengcheng, S.: Meshfree framework for image-derived modelling. In: 2008 5th IEEE International Symposium on Biomedical Imaging: From Nano to Macro, pp. 1449-1452. (2008)

35. A Dassault Systèmes Simulia Corporation: ABAQUS Online Documentation: Version 6.14. (2014) 
36. Chowdhury, H., Joldes, G., Wittek, A., Doyle, B., Pasternak, E., Miller, K.: Implementation of a Modified Moving Least Squares Approximation for Predicting Soft Tissue Deformation Using a Meshless Method. In: Doyle, B., Miller, K., Wittek, A., Nielsen, P.M.F. (eds.) Computational Biomechanics for Medicine, 59-71. Springer International Publishing (2015)

37. Liu, G.R.: Mesh Free Methods: Moving Beyond the Finite Element Method. CRC Press, Boca Raton (2003)

38. Dolbow, J., Belytschko, T.: Numerical integration of the Galerkin weak form in meshfree methods. Computational Mechanics 23, 219-230 (1999)

39. Beissel, S., Belytschko, T.: Nodal integration of the element-free Galerkin method. Computer Methods in Applied Mechanics and Engineering 139, 4974 (1996)

40. Chen, J.-S., Wu, C.-T., Yoon, S., You, Y.: A stabilized conforming nodal integration for Galerkin mesh-free methods. International Journal for Numerical Methods in Engineering 50, 435-466 (2001)

41. Livermore Software Technology Coporation LSTC: LS-DYNA Theory Manual (2017)

42. Quak, W., van den Boogaard, A., González, D., Cueto, E.: A comparative study on the performance of meshless approximations and their integration. Computational Mechanics 48, 121-137 (2011)

43. Wittek, A., Joldes, G., Miller, K.: Algorithms for Computational Biomechanics of the Brain. In: Miller, K. (ed.) Biomechanics of the Brain, 189-219, Springer (2011)

44. Joldes, G.R., Teakle, P., Wittek, A., Miller, K.: Computation of accurate solutions when using element-free Galerkin methods for solving structural problems. Engineering Computations 34, , 902-920 (2017)

45. Mor, A.B., Kanade, T.: Modifying Soft Tissue Models: Progressive Cutting with Minimal New Element Creation. In Delp, Scott L. DiGoia, Anthony M.Jaramaz, Branisla (eds.) Medical Image Computing and Computer-Assisted Intervention - MICCAI 2000, 598-607. Springer Berlin Heidelberg (200)

46. Bielser, D., Glardon, P., Teschner, M., Gross, M.: Interctive simulation of surgical cuts, Graph Models 66, 116-125 (2004)

47. Courtecuisse, H., Jung, H., Allard, J., Duriez, C., Lee, D.Y., Cotin, S.: GPUbased real-time soft tissue deformation with cutting and haptic feedback. Progress in Biophysics and Molecular Biology 103, 159-168 (2010)

48. Courtecuisse, H., Allard, J., Kerfriden, P., Bordas, S., Cotin, S., Duriez, C.: Real-time simulation of contact and cutting of heterogeneous soft-tissues. Medical Image Analysis 18, 394-410 (2014) 
49. Bui, H.P., Tomar, S., Courtecuisse, H., Cotin, S., Bordas, S.P.A.: Real-Time Error Control for Surgical Simulation. IEEE Transactions on Biomedical Engineering 65, 596-607 (2018)

50. Cotin, S., Delingette, H., Ayache, N.: A hybrid elastic model for real-time cutting, deformations, and force feedback for surgery training and simulation. The Visual Computer 16, 437-452 (2000)

51. Belytschko, T., Lu, Y.Y., Gu, L.: Element-free Galerkin methods International Journal for Numerical Methods in Engineering 37, 229-256 (1994)

52. Belytschko, T., Tabbara, M.: Dynamic fracture using element-free Galerkin methods. International Journal for Numerical Methods in Engineering 39, 923-938 (1996)

53. Belytschko, T., Organ, D., Gerlach, C.: Element-free galerkin methods for dynamic fracture in concrete. Computer Methods in Applied Mechanics and Engineering 187, 385-399 (2000)

54. Rabczuk, T., Belytschko, T.: Adaptivity for structured meshfree particle methods in 2D and 3D. International Journal for Numerical Methods in Engineering 63, 1559-1582 (2005)

55. Bordas, S., Rabczuk, T., Zi, G.: Three-dimensional crack initiation, propagation, branching and junction in non-linear materials by an extended meshfree method without asymptotic enrichment. Engineering Fracture Mechanics 75, 943-960 (2008)

56. Ionescu, I., Weiss, J.A., Guilkey, J., Cole, M., Kirby, R.M., Berzins, M.: Ballistic injury simulation using the material point method. Studies in Health Technology and Informatics 119, 228-233 (2006)

57. Rabczuk, T., Belytschko, T.: A three-dimensional large deformation meshfree method for arbitrary evolving cracks. Computer Methods in Applied Mechanics and Engineering 196, 2777-2799 (2007)

58. Belytschko, T., Krongauz, Y., Organ, D., Flaming, M., Krysl, P.: Meshless methods: An overview and recent developments. Computer Methods in Applied Mechanics and Engineering 139, 3-47 (1996)

59. Osher, S., Sethian, J.A.: Fronts propagating with curvature-dependent speed: Algorithms based on Hamilton-Jacobi formulations. Journal of Computational Physics 79, 12-49 (1988)

60. Stolarska, M., Chopp, D.L., Moës, N., Belytschko, T.: Modelling crack growth by level sets in the extended finite element method. International Journal for Numerical Methods in Engineering 51, 943-960 (2001) 
61. Jin, X., Joldes, G.R., Miller, K., Wittek, A.: 3D Algorithm for simulation of soft tissue cutting. In: Wittek, A., Miller, K., Nielsen, P.M.F. (eds.) Computational Biomechanics for Medicine: Models, Algorithms and Implementation, pp. 49-62. Springer, New York, ISBN 978-14614-6350-4 (2013)

62. Gültekin, O., Dal, H., Holzapfel, G.A.: A phase-field approach to model fracture of arterial walls: Theory and finite element analysis. Computer Methods in Applied Mechanics and Engineering 312, 542-566 (2016)

63. Belytschko, T., Liu, W.K., Moran, B.: Nonlinear Finite Elements for Continua and Structures. John Wiley\&Sons, Chichester (2006)

64. Isaacson, E.: Analysis of Numerical Methods. Wiley, New York (1966)

65. Cook, R.D., Malkus, D.S., Plesha, M.E.: Finite elements in dynamics and vibrations. Concepts and Applications of Finite Element Analysis, 367-428. John Wiley \& Sons, New York (1989)

66. Joldes, G.R., Wittek, A., Miller, K.: Stable time step estimates for mesh-free particle methods. International Journal for Numerical Methods in Engineering 91, 450-456 (2012)

67. Bathe, K.-J.: Finite Element Procedures. Prentice-Hall (1996)

68. Puso, M.A., Chen, J.S., Zywicz, E., Elmer, W.: Meshfree and finite element nodal integration methods. International Journal for Numerical Methods in Engineering 74, 416-446 (2008)

69. Waldron, K.J., Kinzel, G.L.: Kinematics, dynamics, and design of machinery. John Wiley, New York (1999)

70. Wittek, A., Bourantas, G., Joldes, G.R., Khau, A., Mountris, K., Singh, S., Miller, K.: Meshless method for simulation of needle insertion into soft tissues: Preliminary results. In: Nash, M., Nielsen, P.M.F., Wittek, A., Miller, K., Joldes, G.R. (eds.) Computational Biomechanics for Medicine, 15 pages Springer (2018) - Accepted for publication.

71. Bucholz, R., MacNeil, W., McDurmont, L.: The operating room of the future. Clinical Neurosurgery 51, 228-237 (2004)

72. Rivlin, R.S., Sawyers, K.N.: The Strain-Energy Function for Elastomers. Transactions of the Society of Rheology 20, 545-557 (1976) 